\title{
Avaliação da acuidade visual Snellen
}

\author{
Snellen visual acuity evaluation
}

\author{
Marcio Zapparoli ${ }^{1}$ \\ Fernando Klein ${ }^{2}$ \\ Hamilton Moreira $^{3}$
}

\begin{tabular}{l} 
RESUMO \\
\hline Objetivos: Avaliar a medida de acuidade visual (AV), por meio da tabela \\
de Snellen, em consultórios de três grandes centros oftalmológicos de \\
Curitiba, comparar essas medidas entre si, com um método computado- \\
rizado para aferição de AV, e também, com o padrão teórico desenvolvido \\
por Hermann Snellen. Métodos: Estudo descritivo incluindo 15 consul- \\
tórios oftalmológicos e um método computadorizado para a medida de \\
AV. Com o auxílio de uma trena e um paquímetro foi realizada a medida \\
da distância do olho do paciente sentado até a parede de projeção dos \\
optotipos e, ao mesmo tempo, medido o tamanho da letra que era \\
projetada na parede para 4 linhas de AV. Resultados: Para a análise dos \\
resultados, optamos por comparar os ângulos apresentados por cada con- \\
sultório no momento da realização da medida. Sem surpresas, observou- \\
se que nenhum consultório obteve o ângulo exato de 5 minutos de arco. \\
Comparando-se os consultórios com o método computadorizado, obser- \\
vou-se que apenas 2 consultórios obtiveram melhores resultados se \\
comparados os ângulos de projeção dos optotipos, porém os dados são \\
muito próximos e insignificantes estatisticamente. Já em relação aos demais \\
consultórios, a superioridade do método computadorizado é bastante \\
relevante. Todos tiveram diferenças significantes em relação ao padrão- \\
ouro. Ainda individualmente, comparou-se o método computadorizado com \\
o padrão teórico Snellen e observou-se que esse obteve condições muito \\
próximas do ideal, ou seja, em todas as acuidades visuais analisadas \\
esteve sempre próximo dos 5 minutos de arco. Conclusões: Podemos \\
concluir que existe uma grande heterogeneidade na avaliação da AV nos \\
diferentes centros de oftalmologia. Também se pode esperar que para um \\
mesmo paciente, avaliado em diferentes consultórios, há a possibilidade \\
de se obter diferenças nas medidas da AV. O método computadorizado \\
avaliado é prático, portátil, ajustável a distâncias diversas, mantém a \\
razão de proporcionalidade dos optotipos baseada no ângulo visual e se \\
aproxima em muito ao padrão teórico preconizado por Herman Snellen \\
em 1862.
\end{tabular}

Descritores: Acuidade visual; Visão; Técnicas de diagnóstico oftalmológico

\section{INTRODUÇ̃̃O}

Os dados históricos mais antigos encontrados sobre a medida da visão indicam que Kuechler, em 1843, um oftalmologista alemão, desenvolveu três tabelas de medida, mas seu trabalho foi esquecido quase que completamente. Jaeger, em 1854, publicou em Viena uma tabela de leitura para documentar a visão, usada por muitos ainda hoje. Donders, em 1861, inventou o termo "acuidade visual" (AV) para descrever a qualidade da visão humana. Sua tabela foi a primeira cientificamente embasada e ficou 
conhecida como o "E" de Donders, que introduziu a unidade de 1 minuto de arco como o ângulo de menor resolução visível para o olho humano. Em 1862, o oftalmologista holandês Herman Snellen, com a ajuda de Donders, publicou sua famosa tabela baseada e definida em "optotipos". Snellen arbitrariamente definiu a "visão padrão" como a habilidade de reconhecer um de seus optotipos com tamanho angular de 5 minutos de arco, sendo o optotipo formado por linhas de espessura e espaçamento de 1 minuto de $\operatorname{arco}^{(1)}$.

A tabela criada por Snellen é o método universalmente aceito para medir a $\mathrm{AV}$, apesar de sua baixa confiabilidade e reprodutibilidade. Nesta tabela algumas letras são mais legíveis do que outras; por exemplo, o "L" é mais fácil de ler do que o "E" e o paciente deve saber ler. Além disso, as tabelas de Snellen têm também o defeito de apresentarem diferentes números de letras em cada linha, o que provoca o fenômeno de agrupamento e espaçamento desproporcional entre as letras e as linhas, além do universo medido não ser suficiente em casos de baixa acuidade visual ${ }^{(1-2)}$.

Desde Snellen, poucas melhorias na medida da acuidade visual foram feitas. Landolt, em 1888, propôs uma interessante modificação, com optotipos circulares anelados, com somente um elemento de quebra, com variação em sua orientação. Assim os pacientes poderiam identificar a orientação de uma lacuna nos anéis mesmo sem saber ler ${ }^{(1,3)}$.

Apenas quase um século após Snellen, estudos atingiram sua proposta inicial. Sloan em 1959, Hyvärinen em 1976, Taylor em 1976, Bailey e Lovie em 1976 e os Protocolos Early Treatment Diabetic Retinopathy Study (EDTRS) em 1991 e International Council of Ophthalmology (ICO) em 2002 conseguiram consenso e padronizaram mundialmente a medida subjetiva da acuidade visual. Estandardizou-se uma tabela com cinco letras em cada fileira iguais ao tamanho da letra. Desta maneira, o efeito aglomerado e o número dos erros que poderiam ser cometidos em cada linha transformaram o tamanho da letra a única variável entre os níveis de acuidade visual medidos ${ }^{(1,4)}$.

O presente estudo objetiva avaliar e comparar os principais consultórios de três centros oftalmológicos de Curitiba entre si utilizando-se a tabela de Snellen, que é o método padrão na medida da $\mathrm{AV}$, comparar estes consultórios com o método computadorizado desenvolvido para a medida da acuidade visual e, por último, o padrão teórico idealizado por Snellen com o método computadorizado.

\section{MÉTODOS}

Este projeto de pesquisa foi submetido à avaliação de um Comitê de Ética em pesquisa, tendo sido aprovado em 09/03/ 2009.

O estudo foi realizado em três grandes centros de oftalmologia da cidade de Curitiba e envolveu a participação de 8 consultórios do Hospital de Olhos do Paraná, 4 consultórios do serviço de residência da Universidade Federal do Paraná e 3 consultórios do serviço de residência do Hospital Universitário
Evangélico de Curitiba. Esses consultórios foram escolhidos por representarem a maior demanda de pacientes no serviço de oftalmologia em Curitiba.

O método computadorizado utilizado para comparação com o padrão Snellen foi escolhido com base em um modelo utilizado recentemente em alguns centros. Trata-se de um sistema computadorizado para avaliação da acuidade visual que utiliza display LCD de 17", iluminação de fundo de $200 \mathrm{~cd} / \mathrm{m}^{2}$ e escala de refração ajustável de 2,0 a 6,0 m. Além da aferição da AV, esse sistema possui diversas outras funções tais como teste de sensibilidade ao contraste, teste de cores Ishihara, " $C$ " de Landolt, cilindro cruzado, optotipos para crianças, teste de letras, "E" de Snellen, números, ETDRS, HOVT, luzes de Worth além de possibilitar a reprodução de vídeos entre outras. Porém, esse estudo visa avaliar somente o teste de AV.

$\mathrm{Na}$ primeira etapa do estudo foram determinados os padrões teóricos de optotipos para a AV de 20/15, 20/20, 20/40 e 20/100 idealizados por Snellen. O tamanho dos optotipos foi calculado com base no ângulo visual de 5 minutos de arco, formado no ponto nodal do olho quando visto a 15, 20, 40 e 100 pés respectivamente. Procedimento este que nos levou a determinação da tabela de Snellen e consequentemente ao tamanho correspondente de cada optotipo de cada linha de visão. Esse é o método consagrado como padrão-ouro para a medida da acuidade visual na prática clínica.

$\mathrm{Na}$ sequência todos os consultórios foram visitados e, com o auxílio de uma trena métrica de 7,5 m da marca Feeling e um paquímetro de $150 \mathrm{~mm}$ com escala de $0,05 \mathrm{~mm}$ da marca Vonder, foi realizada a medida da distância do olho do paciente sentado até a parede de projeção dos optotipos e, ao mesmo tempo, medido o tamanho da letra que era projetada na parede para cada linha de AV de 20/15, 20/20, 20/40 e 20/100. Além disso, levamos em consideração a marca de cada projetor utilizado nos diversos consultórios.

$\mathrm{O}$ mesmo procedimento foi realizado sempre na mesma sequência e sempre pelo mesmo examinador. Os dados obtidos eram armazenados em banco de dados para posterior comparação e análise.

Como nos interessa apenas a avaliação da medida de acuidade visual e, também, por motivos de ética, os consultórios foram agrupados sem distinção de qual centro oftalmológico pertenciam e sem referência ao profissional atuante neles. Segue abaixo a disposição dos dados na tabela 1 para melhor visualização e compreensão.

A segunda etapa do estudo compreendeu a determinação dos valores teóricos para cada consultório, levando-se em consideração que a distância de onde o paciente está sentado até a parede de projeção dos optotipos é diferente para cada um e que, portanto, cada consultório teria uma razão de proporcionalidade no tamanho da letra projetada na parede em comparação com o preconizado por Herman Snellen em 1862.

Esses dados foram úteis apenas para compreendermos como deveria ser a aferição da acuidade visual Snellen, nos consultórios, caso eles apresentassem exatamente a projeção dos optotipos no ângulo correto de 5 minutos de arco e respei- 


\begin{tabular}{|c|c|c|c|c|c|c|}
\hline \multirow[b]{2}{*}{ Consultório } & \multicolumn{4}{|c|}{ AV (projeção dos optotipos) } & \multirow{2}{*}{$\begin{array}{c}\text { Distância do } \\
\text { paciente até } \\
\text { a projeção }\end{array}$} & \multirow{2}{*}{$\begin{array}{l}\text { Projetor } \\
\text { utilizado }\end{array}$} \\
\hline & $20 / 15$ & $20 / 20$ & $20 / 40$ & $20 / 100$ & & \\
\hline Consultório 1 & $7,65 \mathrm{~mm}$ & $10,60 \mathrm{~mm}$ & $21,60 \mathrm{~mm}$ & $52,75 \mathrm{~mm}$ & $5,06 \mathrm{~m}$ & Xenônio \\
\hline Consultório 2 & $7,15 \mathrm{~mm}$ & $9,35 \mathrm{~mm}$ & $14,00 \mathrm{~mm}$ & $49,00 \mathrm{~mm}$ & $4,88 \mathrm{~m}$ & Xenônio \\
\hline Consultório 3 & $4,45 \mathrm{~mm}$ & $6,35 \mathrm{~mm}$ & $13,50 \mathrm{~mm}$ & $36,15 \mathrm{~mm}$ & $4,70 \mathrm{~m}$ & Xenônio \\
\hline Consultório 4 & $5,25 \mathrm{~mm}$ & $7,15 \mathrm{~mm}$ & $14,65 \mathrm{~mm}$ & $35,40 \mathrm{~mm}$ & $4,03 \mathrm{~m}$ & Xenônio \\
\hline Consultório 5 & $5,70 \mathrm{~mm}$ & $7,00 \mathrm{~mm}$ & $14,25 \mathrm{~mm}$ & $37,15 \mathrm{~mm}$ & $4,73 \mathrm{~m}$ & Reichert \\
\hline Consultório 6 & $5,55 \mathrm{~mm}$ & $7,25 \mathrm{~mm}$ & $14,45 \mathrm{~mm}$ & $36,35 \mathrm{~mm}$ & $5,00 \mathrm{~m}$ & Eyetec \\
\hline Consultório 7 & $5,00 \mathrm{~mm}$ & $6,00 \mathrm{~mm}$ & $12,55 \mathrm{~mm}$ & $32,00 \mathrm{~mm}$ & $3,87 \mathrm{~m}$ & Xenônio \\
\hline Consultório 8 & $5,30 \mathrm{~mm}$ & $7,45 \mathrm{~mm}$ & $14,00 \mathrm{~mm}$ & $34,55 \mathrm{~mm}$ & $3,47 \mathrm{~m}$ & Oftalmo-tec \\
\hline Consultório 9 & $3,65 \mathrm{~mm}$ & $4,45 \mathrm{~mm}$ & $9,00 \mathrm{~mm}$ & $22,70 \mathrm{~mm}$ & $3,55 \mathrm{~m}$ & SZP 350 \\
\hline Consultório 10 & $3,35 \mathrm{~mm}$ & $4,30 \mathrm{~mm}$ & $8,35 \mathrm{~mm}$ & $21,25 \mathrm{~mm}$ & $3,41 \mathrm{~m}$ & Zeiss \\
\hline Consultório 11 & $3,70 \mathrm{~mm}$ & $4,70 \mathrm{~mm}$ & $9,35 \mathrm{~mm}$ & $23,35 \mathrm{~mm}$ & $3,30 \mathrm{~m}$ & Zeiss \\
\hline Consultório 12 & $3,60 \mathrm{~mm}$ & $4,60 \mathrm{~mm}$ & $9,00 \mathrm{~mm}$ & $22,45 \mathrm{~mm}$ & $3,29 \mathrm{~m}$ & Zeiss \\
\hline Consultório 13 & $4,45 \mathrm{~mm}$ & $5,60 \mathrm{~mm}$ & $11,45 \mathrm{~mm}$ & $29,35 \mathrm{~mm}$ & $3,43 \mathrm{~m}$ & Bausch \& Lomb \\
\hline Consultório 14 & $4,45 \mathrm{~mm}$ & $5,65 \mathrm{~mm}$ & $12,00 \mathrm{~mm}$ & $31,30 \mathrm{~mm}$ & $3,85 \mathrm{~m}$ & Bausch \& Lomb \\
\hline Consultório 15 & - & $7,95 \mathrm{~mm}$ & $13,35 \mathrm{~mm}$ & $31,90 \mathrm{~mm}$ & $3,79 \mathrm{~m}$ & Bausch \& Lomb \\
\hline
\end{tabular}

tando-se o padrão de proporcionalidade referente à distância do paciente até a projeção disponível em cada consultório. A tabela 2 nos mostra, em detalhes, o que se esperava enquanto se realizava a medição.

A terceira e última etapa deste trabalho envolveu a determinação do ângulo real de projeção do optotipo em cada consultório analisado. Para a determinação desse ângulo de projeção do optotipo de cada consultório utilizou-se a função trigonométrica arco tangente, sendo que os dados coletados na primeira etapa foram utilizados. Considerou-se no cálculo o valor medido do optotipo projetado da AV 20/20 por ser o padrão de acuidade visual preconizado normal.

Na sequência, de posse do ângulo real calculado, os valores teóricos para a AV 20/15, 20/20, 20/40 e 20/100 puderam ser determinados com base no ângulo real que cada consultório apresentava (Tabela 3)

Por fim, foram calculados a média, desvio padrão, valor máximo e valor mínimo das medidas armazenadas dos consultórios de cada centro oftalmológico separadamente com objetivo de facilitar a análise da aferição da AV em comparação ao padrão teórico Snellen.

\section{RESULTADOS}

Para a análise dos resultados, optamos por comparar os ângulos apresentados por cada consultório no momento da realização da medida. Sem surpresas, observou-se que nenhum consultório obteve o ângulo exato de 5 minutos de arco. Todos tiveram diferenças significantes em relação ao padrão-ouro.

Dentre os melhores pudemos destacar os consultórios 5, 6 e 14. Estes tiveram ângulos de projeção respectivamente a 5,08 minutos de arco, 4,97 minutos de arco e 5,03 minutos de arco, ou seja, muito próximo do ideal. Já entre os piores destacaram-se os consultórios 1, 8 e 15 que obtiveram ângulos de 7,17 minutos de arco, 7,34 minutos de arco e 7,18 minutos de arco respectivamente, ou seja, muito além do que se esperava (Gráfico 1). O que pode ser entendido como uma AV superestimada. Por exemplo, se considerarmos a medida da AV de um paciente no consultório 8, erroneamente estaremos registrando sua visão como 20/20 e, no entanto, esse mesmo paciente não estará apto a ler esta mesma linha em um consultório adequado ao padrão Snellen.

Comparando-se os consultórios com o método computadorizado, observou-se que apenas os consultórios 6 e 14 obtiveram melhores resultados se comparados os ângulos de projeção dos optotipos, porém os dados são muito próximos e insignificantes estatisticamente. Já em relação aos demais consultórios, a superioridade do método computadorizado é bastante relevante.

Ainda individualmente, comparou-se o método computadorizado com o padrão teórico Snellen e observou-se que esse obteve condições muito próximas do ideal, ou seja, em todas as acuidades visuais analisadas esteve sempre próximo dos 5 minutos de arco.

Coletivamente dividimos os 3 centros de oftalmologia para entendermos como eles se comportavam em grupos. No geral o grupo 1 obteve como média dos consultórios um ângulo de 5,894 minutos de arco, enquanto os grupos 2 e 3 obtiveram 4,58 minutos de arco e 5,94 minutos de arco respectivamente (Gráfico 2).

Analisando-se por grupo, individualmente cada consultório, concluímos que o grupo 1 foi o que apresentou o consultório que mais se distanciou do padrão Snellen, com um valor de 7,34 minutos de arco, contra 7,18 minutos de arco de um dos consultórios do grupo 3 e 4,89 minutos de arco de um dos consultórios do grupo 2. Levando-se em conta o valor mínimo, os dados se invertem. O grupo 2 foi o que apresentou o consultório com menor valor medido em 4,30 minutos de arco, contra 4,64 minutos de arco de um dos consultórios do grupo 1 e 5,03 minutos de arco de um dos consultórios do grupo 3 . 


\begin{tabular}{|c|c|c|c|c|}
\hline \multirow[b]{2}{*}{ Consultório } & \multicolumn{4}{|c|}{ AV (projeção dos optotipos) } \\
\hline & $20 / 15$ & $20 / 20$ & $20 / 40$ & $20 / 100$ \\
\hline Consultório 1 & $5,52 \mathrm{~mm}$ & $7,36 \mathrm{~mm}$ & $14,72 \mathrm{~mm}$ & $36,80 \mathrm{~mm}$ \\
\hline Consultório & $5,32 \mathrm{~mm}$ & $7,10 \mathrm{~mm}$ & $14,20 \mathrm{~mm}$ & $35,49 \mathrm{~mm}$ \\
\hline Consultório 3 & $5,13 \mathrm{~mm}$ & $6,84 \mathrm{~mm}$ & $13,67 \mathrm{~mm}$ & $34,18 \mathrm{~mm}$ \\
\hline Consultório & $4,40 \mathrm{~mm}$ & $5,86 \mathrm{~mm}$ & $11,72 \mathrm{~mm}$ & $29,31 \mathrm{~mm}$ \\
\hline Consultório 5 & $5,16 \mathrm{~mm}$ & $6,88 \mathrm{~mm}$ & $13,76 \mathrm{~mm}$ & $34,40 \mathrm{~mm}$ \\
\hline Consultório & $5,45 \mathrm{~mm}$ & $7,27 \mathrm{~mm}$ & $14,54 \mathrm{~mm}$ & $36,36 \mathrm{~mm}$ \\
\hline Consultório & $4,22 \mathrm{~mm}$ & $5,63 \mathrm{~mm}$ & $11,26 \mathrm{~mm}$ & $28,14 \mathrm{~mm}$ \\
\hline Consultório & $3,79 \mathrm{~mm}$ & $5,05 \mathrm{~mm}$ & $10,09 \mathrm{~mm}$ & $25,23 \mathrm{~mm}$ \\
\hline Consultório 9 & $3,87 \mathrm{~mm}$ & $5,16 \mathrm{~mm}$ & $10,33 \mathrm{~mm}$ & $25,82 \mathrm{~mm}$ \\
\hline Consultório 10 & $3,72 \mathrm{~mm}$ & $4,96 \mathrm{~mm}$ & $9,92 \mathrm{~mm}$ & $24,80 \mathrm{~mm}$ \\
\hline Consultório 11 & $3,60 \mathrm{~mm}$ & $4,80 \mathrm{~mm}$ & $9,60 \mathrm{~mm}$ & $24,00 \mathrm{~mm}$ \\
\hline Consultório 12 & $3,59 \mathrm{~mm}$ & $4,79 \mathrm{~mm}$ & $9,57 \mathrm{~mm}$ & $23,93 \mathrm{~mm}$ \\
\hline Consultório 13 & $3,74 \mathrm{~mm}$ & $4,99 \mathrm{~mm}$ & $9,98 \mathrm{~mm}$ & $24,94 \mathrm{~mm}$ \\
\hline Consultório 14 & $4,20 \mathrm{~mm}$ & $5,60 \mathrm{~mm}$ & $11,20 \mathrm{~mm}$ & $28,00 \mathrm{~mm}$ \\
\hline Consultório 15 & - & $5,51 \mathrm{~mm}$ & $11,02 \mathrm{~mm}$ & $27,56 \mathrm{~mm}$ \\
\hline
\end{tabular}

\begin{tabular}{|c|c|c|c|c|c|}
\hline \multirow[b]{2}{*}{ Consultório } & \multirow{2}{*}{$\begin{array}{l}\text { Ângulo de } \\
\text { projeção real }\end{array}$} & \multicolumn{4}{|c|}{ AV (projeção dos optotipos) } \\
\hline & & $20 / 15$ & $20 / 20$ & $20 / 40$ & $20 / 100$ \\
\hline Consultório 1 & 7,17 arc $\min$ & $9,58 \mathrm{~mm}$ & $12,80 \mathrm{~mm}$ & $25,50 \mathrm{~mm}$ & $63,90 \mathrm{~mm}$ \\
\hline Consultório 2 & 6,56 arc $\min$ & $8,76 \mathrm{~mm}$ & $11,70 \mathrm{~mm}$ & $23,40 \mathrm{~mm}$ & $58,40 \mathrm{~mm}$ \\
\hline Consultório & 4,64 arc $\min$ & $6,18 \mathrm{~mm}$ & $8,24 \mathrm{~mm}$ & $16,50 \mathrm{~mm}$ & $41,20 \mathrm{~mm}$ \\
\hline Consultório & 6,08 arc $\min$ & $6,11 \mathrm{~mm}$ & $10,80 \mathrm{~mm}$ & $21,60 \mathrm{~mm}$ & $54,10 \mathrm{~mm}$ \\
\hline Consultório & 5,08 arc $\min$ & $6,77 \mathrm{~mm}$ & $9,02 \mathrm{~mm}$ & $18,00 \mathrm{~mm}$ & $45,10 \mathrm{~mm}$ \\
\hline Consultório & 4,97 arc $\min$ & $6,63 \mathrm{~mm}$ & $8,84 \mathrm{~mm}$ & $17,70 \mathrm{~mm}$ & $44,20 \mathrm{~mm}$ \\
\hline Consultório & 5,32 arc $\min$ & $7,09 \mathrm{~mm}$ & $9,45 \mathrm{~mm}$ & $18,90 \mathrm{~mm}$ & $47,30 \mathrm{~mm}$ \\
\hline Consultório & 7,34 arc min & $9,82 \mathrm{~mm}$ & $13,10 \mathrm{~mm}$ & $26,20 \mathrm{~mm}$ & $65,40 \mathrm{~mm}$ \\
\hline Consultório 9 & 4,30 arc $\min$ & $5,73 \mathrm{~mm}$ & $7,64 \mathrm{~mm}$ & $15,30 \mathrm{~mm}$ & $38,20 \mathrm{~mm}$ \\
\hline Consultório 10 & 4,33 arc min & $5,77 \mathrm{~mm}$ & $7,69 \mathrm{~mm}$ & $15,40 \mathrm{~mm}$ & $38,40 \mathrm{~mm}$ \\
\hline Consultório 11 & 4,89 arc $\min$ & $6,51 \mathrm{~mm}$ & $8,68 \mathrm{~mm}$ & $17,40 \mathrm{~mm}$ & $43,40 \mathrm{~mm}$ \\
\hline Consultório 12 & 4,80 arc $\min$ & $6,39 \mathrm{~mm}$ & $8,52 \mathrm{~mm}$ & $17,00 \mathrm{~mm}$ & $42,60 \mathrm{~mm}$ \\
\hline Consultório 13 & 5,60 arc $\min$ & $7,46 \mathrm{~mm}$ & $9,95 \mathrm{~mm}$ & $19,90 \mathrm{~mm}$ & $49,80 \mathrm{~mm}$ \\
\hline Consultório 14 & 5,03 arc min & $6,71 \mathrm{~mm}$ & $8,95 \mathrm{~mm}$ & $17,90 \mathrm{~mm}$ & $44,70 \mathrm{~mm}$ \\
\hline Consultório 15 & 7,18 arc $\min$ & $9,59 \mathrm{~mm}$ & $12,80 \mathrm{~mm}$ & $25,60 \mathrm{~mm}$ & $63,90 \mathrm{~mm}$ \\
\hline
\end{tabular}

Comparando-se cada centro oftalmológico observou-se que o grupo 2 obteve um desvio padrão bem abaixo dos demais com

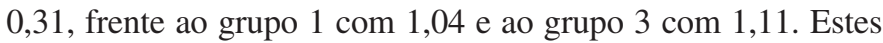
dados podem nos sugerir que o grupo 2 é o mais homogêneo em relação aos demais grupos.

\section{DISCUSS ÃO}

A medida da acuidade visual é o teste mais comumente utilizado para a determinação da função visual na prática oftalmológica e, embora a técnica de exame seja simples, o processo utilizado é complexo e exige a interação de muitos fatores, tanto fisiológicos quanto psicológicos. A avaliação da acuidade visual requer que o olho detecte o objeto e faça distinção entre seus componentes. Essa informação é, então, transmitida ao córtex cerebral, onde é comparada com as formas existentes na memória. O paciente deve ser capaz de comunicar o reconhecimento do objeto ao médico. Fisiologicamente, a AV é determinada pela habilidade de distinguir dois estímulos separados no espaço em contraste com o fundo. Esta simples medida detecta grande parte das disfunções visuais, sendo assim sua correta aferição é um importante método semiológico na prática oftalmológica ${ }^{(1,4-5)}$.

Cada consultório oftalmológico possui suas peculiaridades, distância da cadeira até a tela de projeção, iluminação, cores, modelo de projetor, não existindo um padrão específico, e nem sempre as suas medidas são adequadas para proporcionar o ângulo visual idealizado por Herman Snellen, considerado o padrão-ouro atual. Além disso, como se sabe, a função visual é um sentido muito mais amplo e difícil de ser avaliado 


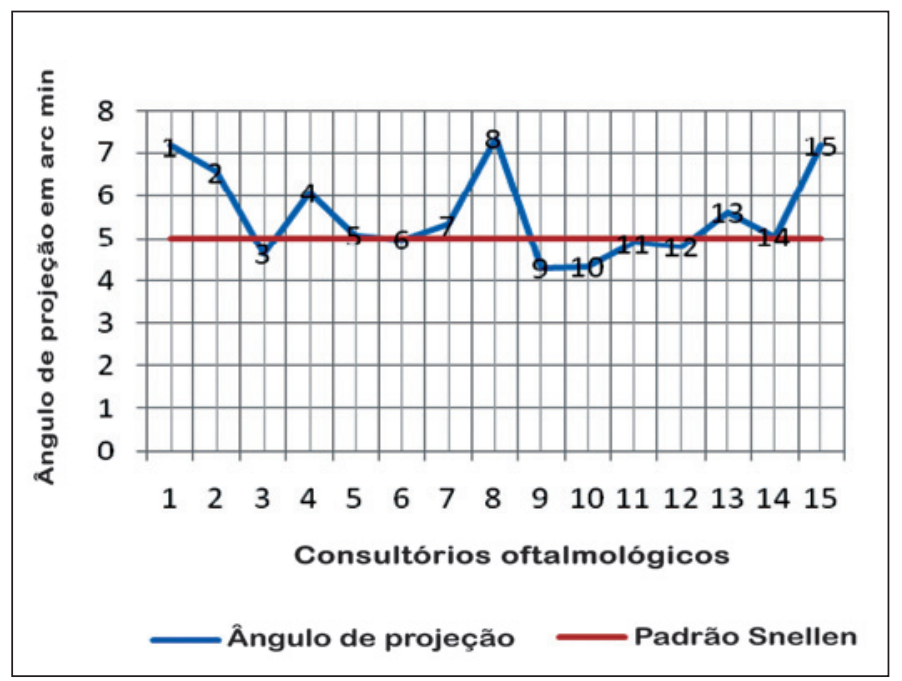

Gráfico 1 - Ângulo de projeção de cada consultório oftalmológico em relação ao padrão Snellen

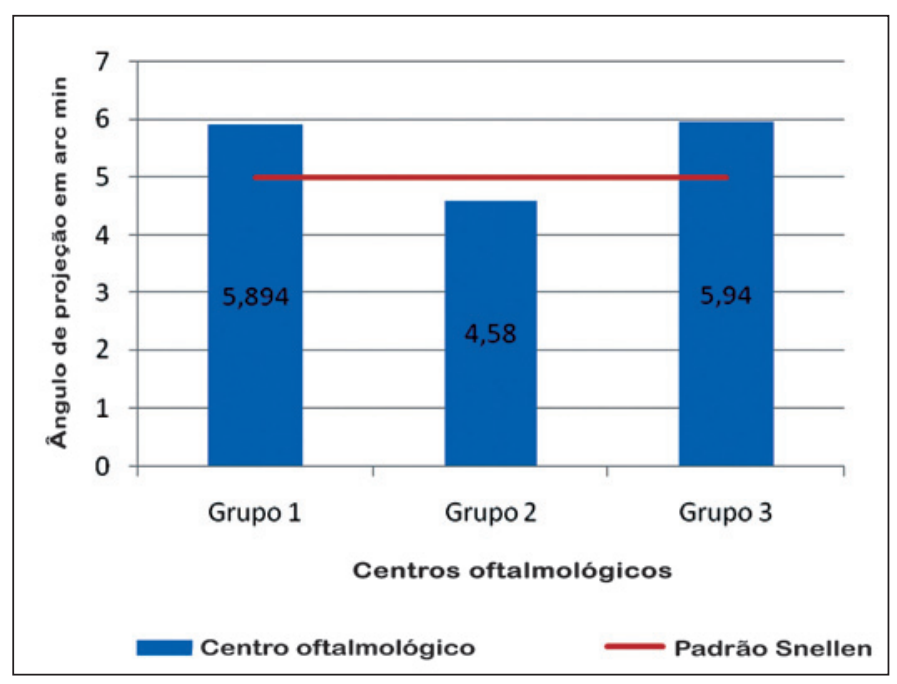

Gráfico 2 - Média dos ângulos de projeção dos consultórios por grupo em relação ao padrão Snellen

do que meramente uma medida numérica de AV. Dessa maneira é virtualmente impossível considerar como exatos os resultados de AV aferidos na grande maioria das clínicas oftalmológicas e esses dados podem e são atualmente utilizados para a prática diária, porém não devem ser interpretados como resultados adequados na produção de trabalhos científicos envolvendo essa variável ${ }^{(6)}$.

Já ficou consagrado que a tabela utilizada no ETDRS em 1991 e posteriormente padronizada no International Council of Ophthalmology em 2002 é a melhor forma de se interpretar de maneira correta a variável da AV em produções científicas. Entretanto, é uma tabela muito menos comum e prática de ser utilizada. A partir do momento em que se desenvolvam métodos novos, quer sejam computadorizados ou não, que possam avaliar com mais exatidão e de maneira mais completa a variável
$\mathrm{AV}$, que tenham boa reprodutibilidade, que sejam de baixo custo e de acesso universal, os resultados poderão ser empregados em produções científicas e também serão muito mais confiáveis no dia-a-dia do médico oftalmologista ${ }^{(7)}$.

\section{CONCLUSÃO}

Podemos concluir que existe uma grande heterogeneidade na avaliação da AV nos diferentes centros de oftalmologia. São raros os consultórios onde exista distância suficiente e adequada a tabela de projeção, levando-se em consideração os 20 pés propostos por Snellen. Também são muito raros os locais onde sejam implementados jogo de espelhos para se obter o ângulo visual adequado e minimizar o fenômeno acomodação.

Também se pode esperar que para um mesmo paciente, avaliado em diferentes consultórios, há a possibilidade de se obter diferenças nas medidas da AV.

O método computadorizado avaliado é prático, portátil, ajustável a distâncias diversas, mantém a razão de proporcionalidade dos optotipos baseada no ângulo visual e se aproxima em muito ao padrão teórico preconizado por Herman Snellen em 1862.

Serão precisos estudos futuros, com o emprego de testes de AV em pacientes para podermos avaliar e confirmar a reprodutibilidade e diferenças deste método em comparação aos diversos consultórios e também à tabela estandardizada pelo ETDRS.

\section{ABSTRACT}

Purpose: To evaluate the visual acuity (VA) measurement by Snellen chart at three main Ophthalmology centers in Curitiba, compare these measurements with a computerized method for calibrating VA and also with the standard theory developed by Hermann Snellen. Methods: Descriptive study including 15 Ophthalmology clinics and a computerized method to measure the VA. Using a tape measure and a caliper, measurement of the distance was obtained from the sitting patients' eye to VA optotypes projection on the wall. At the same time, measurement of the size of the letters of 4 lines of the VA projection on the wall was performed. Results: To analyze the results, it was opted to compare the angles presented by each clinic for each measurement. It was not surprising that no clinic presented an exact angle of 5 arch minutes. Comparing clinics results with computerized method, we observed that only 2 clinics obtained better results if compared with the angles obtained from optotypes projection, but the data is very similar and with no statistical significance. In relation to the other clinics, the superiority of the computerized method is very relevant. All had differences in comparison to the gold standard. Individually, com- 
paring computerized method to standard Snellen theory, it was observed that a condition very close to the ideal was obtained, in other words, results very similar to 5 arch minutes were noted in all the visual acuities analyzed. Conclusions: It can be concluded that there is a great heterogeneity in the evaluation of VA in different Ophthalmology centers. Also, the same patient, evaluated in different clinics, can obtain different VA measurements. The computerized method is practical, portable, adjustable for different distances, and maintains optotypes proportionality ratio based on visual angle and approaches the theoretical values recommended by Herman Snellen in 1862.

Keywords: Visual acuity; Vision; Diagnostic techniques, ophthalmological

\section{REFERÊNCIAS}

1. Kronbauer AL, Schor P, Carvalho LAV. Medida da visão e testes psicofísicos. Arq Bras Oftalmol. 2008;71(1):122-7.

2. Falkenstein IA, Cochran DE, Azen SP, Dustin L, Tammewar AM, Kozak I, Freeman WR. Comparison of visual acuity in macular degeneration patients measured with Snellen and early treatment diabetic retinopathy study charts. Ophthalmology. 2008;115(2):319-23.

3. Kniestedt C, Stamper RL. Visual acuity and its measurement. Ophthalmol Clin North Am. 2003;16(2):155-70, v.

4. Beck RW, Moke PS, Turpin AH, Ferris FL $3^{\text {rd }}$, SanGiovanni JP, Johnson CA, et al. A computerized method of visual acuity testing: adaptation of the early treatment of diabetic retinopathy study testing protocol. Am J Ophthalmol. 2003; 135(2):194-205.

5. Spalton DJ, Hitchings RA, Hunter PA. Atlas de oftalmologia clínica. $3^{\mathfrak{a}}$ ed. Rio de Janeiro: Elsevier; 2006.

6. Hussain B, Saleh GM, Sivaprasad S, Hammond CJ. Changing from Snellen to LogMAR: debate or delay? Clin Experiment Ophthalmol. 2006;34(1):6-8.

7. Arippol PKK, Salomão SR, Belfort Júnior R, Método computadorizado para medida da acuidade visual. Arq Bras Oftalmol. 2006;69(6):907-14. 